\section{Interpreting the folding kinetics of helical proteins}

Yaoqi Zhou ${ }^{\star}$ \& Martin Karplus ${ }^{\star} \dagger$

* Department of Chemistry \& Chemical Biology, Harvard University, Cambridge, Massachusetts 02138, USA

$\dagger$ Laboratoire de Chimie Biophysique, ISIS, Université Louis Pasteur, 67000 Strasbourg, France

The detailed mechanism of protein folding is one of the major problems in structural biology ${ }^{1,2}$. Its solution is of practical as well as fundamental interest because of its possible role in utilizing the many sequences becoming available from genomic analysis ${ }^{3}$. Although the Levinthal paradox ${ }^{4}$ (namely, that a polypeptide chain can find its unique native state in spite of the astronomical number of configurations in the denatured state) has been resolved $^{4-7}$, the reasons for the differences in the folding behaviour of individual proteins remain to be elucidated. Here a $\mathrm{C}_{\alpha^{-}}$ based three-helix-bundle-like protein model with a realistic thermodynamic phase diagram is used to calculate several hundred folding trajectories. By varying a single parameter, the difference between the strength of native and non-native contacts, folding is changed from a diffusion-collision mechanism ${ }^{8}$ to one that involves simultaneous collapse and partial secondary-structure formation, followed by reorganization to the native structure. Non-obligatory intermediates are important in the former, whereas there is an obligatory on-pathway intermediate in the latter. Our results provide a basis for understanding the range of folding behaviour that is observed in helical proteins.

All-atom models with explicit solvent have provided information on high-temperature unfolding ${ }^{9,10}$ and the free-energy landscapes of

a

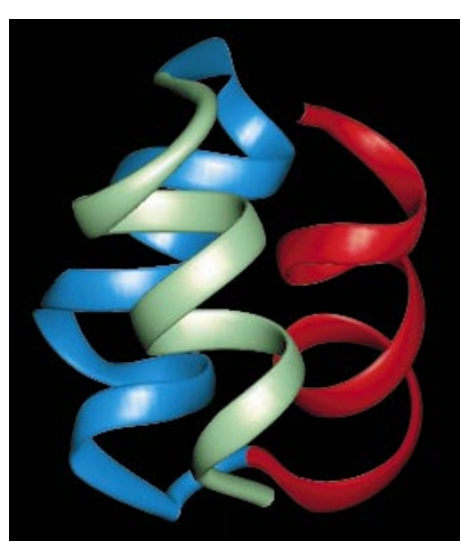

b

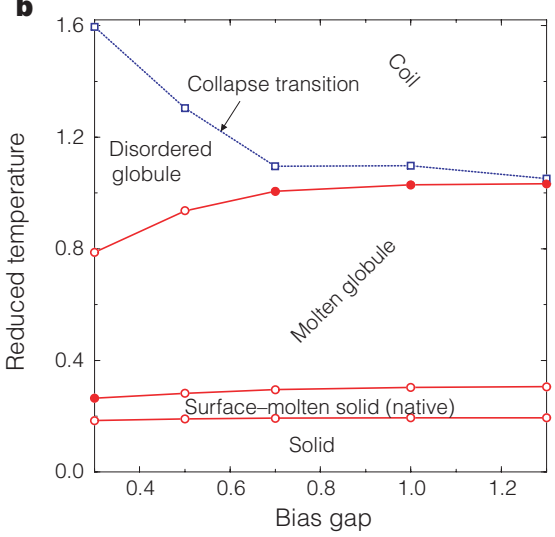

several proteins ${ }^{11,12}$. However, corresponding simulations of the folding kinetics for ensembles of trajectories are beyond what can be done with available computers ${ }^{13}$, as folding times are milliseconds or longer for most proteins. To overcome such computational limitations, we use discrete molecular dynamics to study the folding of the small (46-residue) three-helix-bundle fragment of protein A (ref. 14; and Fig. 1a). The thermodynamic behaviour of the model (Fig. 1b) is in accord with simulations of crambin using an all-atom representation and the temperature-dependent X-ray diffraction results for ribonuclease A (ref. 15), as well as data for other proteins ${ }^{16,17}$. An important element of the $\operatorname{model}^{18}$ is that the phase diagram can be varied by the choice of a single parameter, the bias gap (see Methods), which determines the relative stability of native and non-native contacts. For example, equilibrium collapse to a disorganized globule occurs for a small bias gap and to an organized (molten) globule for a large bias gap, as shown in Fig. 1 b. To analyse the folding kinetics, 100 trajectories, each $100 \mu$ s to $1 \mathrm{~ms}$ in length, were calculated for a series of values of the bias gap parameter, starting from the fully denatured state under conditions where the native state is stable (see Methods). The folding times range from $60 \mathrm{~ns}$ to more than $0.1 \mathrm{~ms}$ for the highly optimized model (large bias, gap, $g=1.3$ ) and from $2 \mu$ s to more than $1 \mathrm{~ms}$ for a weakly optimized model $(g=0.3)$.

The range of folding behaviour is illustrated in Fig. 2. The kinetics of two trajectories for a small value of the bias parameter $(g=0.3)$, both are very different, although identical model parameters are used and both reach the native state. The first (Fig. 2a) has early helix formation (that is, about $70 \%$ of the helical contacts exist at $10 \mathrm{~ns}$ ), whereas other progress variables (the fraction of native interhelical contacts and the inverse native fraction of the volume, for example) are still very far from the native state. The resulting near-helical peptide chain then undergoes a slow diffusion-collision-like search ${ }^{8}$ of the relative helix orientations until the native state is reached at $19 \mu \mathrm{s}$. In this folding scheme, there can be

Figure 1 Structure and thermodynamics of the model three-helix bundle protein. a, Global minimum structure of the model obtained by annealing the NMR structure of the three-helix-bundle fragment (residues 10-55) of Staphylococcus aureus protein A (ref. 14); helix I, II and III are shown in green, blue and red, respectively. b, The phase diagram for models with different bias gap values. All temperatures are in units of the energy parameter $\epsilon$. Thermal transitions and collapse transition are indicated by open circles and open squares, respectively. The filled circles indicate a first-order-like two-state transition. There are four significant transitions: a collapse transition; a disordered to ordered (molten) globule transition; a globule to native-state transition; and the transition from the active native state to a frozen inactive state ${ }^{15,22}$ 
intermediates in which two helices are packed roughly as in the native state (for example, I and II), and the third (for example, III) is extended in a structure appropriate for domain-swapped dimer formation $^{19}$ (structure at $1 \mu \mathrm{s}$; Fig. 2a). Such a folding trajectory is rare for small bias gap values, but it dominates if the bias gap is large $(g \geq 1)$. A trajectory corresponding to the predominant mechanism for the small bias gap is shown in Fig. 2b. It involves simultaneous rapid partial helix formation and collapse $(90 \%$ at $t \approx 200 \mathrm{~ns})$ to a globule that has transient formation of about $20 \%$ of the native tertiary contacts, some of which have to be broken to reach the native state. The collapse is followed by a slow $(\sim 500 \mu \mathrm{s})$ search for the native state within the compact globule; the helical content increases to its final value as the correct tertiary structure is formed.

As found in unfolding simulations of all-atom models ${ }^{20}$, the folding of the three-helix-bundle model involves broad ensembles of trajectories, but they can be classified into dominant pathways (Fig. 3). The large-gap (highly optimized) model has a 'fast track' with no intermediates (folding time $\sim 0.1 \mu \mathrm{s}$ ) and slower tracks with intermediates, which require partial unfolding. The kinetic intermediates involve incorrect arrangements of the three helices $\left(I_{1}\right)$ (Fig. 2a) or locally misfolded regions $\left(I_{2}\right)$. In contrast, the smallgap model has an on-pathway intermediate $\left(\mathrm{I}_{1}^{\prime}\right)$ with a wide range of structures, including varying amounts of helix formation (Fig. 2). $\mathrm{I}_{1}^{\prime}$ is an obligatory on-pathway intermediate because it is present in all trajectories, both folded and not folded ${ }^{21}$. 'Fast-track' folding is exponential, whereas the folding with intermediates is non-exponential, as expected.

The long-lived intermediates $\left(\mathrm{I}_{1}, \mathrm{I}_{1}^{\prime}\right.$ and $\left.\mathrm{I}_{2}\right)$ found in the simulations (Fig. 3) involve a distribution of misfolded regions that trap the system. Specifically, the $\mathrm{C}_{\alpha}$ pseudodihedral angles for one or more residues are in the wrong region. A wide range of lifetimes for misfolded intermediates occur in the simulations; they vary from several nanoseconds to longer than the simulation time. The escape generally involves on-site pseudodihedral angle transitions. This indicates that successful folding may be possible only at temperatures that are high enough to permit such transitions ${ }^{15,22}$. The native contacts of the kinetic intermediates $\left(\mathrm{I}_{1}, \mathrm{I}_{1}^{\prime}\right.$ and $\left.\mathrm{I}_{2}\right)$ are in the range of the corresponding equilibrium-disordered and molten globule states, but the former have more non-native contacts.

The collapsed intermediate $\mathrm{I}_{1}^{\prime}$ shown in Fig. $2 \mathrm{~b}$ (structures at 10 and $100 \mu \mathrm{s}$ ) is similar to the metastable intermediate in ref. 13; both have a radius of gyration that is near the native value, and partially formed helical segments $(40-60 \%)$ with approximately the correct positions and several pseudodihedral angles whose signs are opposite to the native values. The present results can be related also to all-atom calculations of the equilibrium free-energy surface for folding of the same three-helix-bundle fragment. There are two sets of populations ${ }^{11,12}$ : in one, helix formation is early (that is, it occurs in the state without collapse); in the other, there is little helical content in the uncollapsed state. These two dominant regions on the free-energy surface correspond to the kinetic behaviour of the weakly optimized off-lattice model.

The complete description of the folding kinetics and thermodynamics of this model system suggests some general conclusions concerning the folding mechanisms of helical proteins. In particular, a protein that is optimized for fast folding has its rate limited by the collapse transition; in other words, the intrahelical (secondary) contacts can be formed rapidly, even in the absence of collapse, and interhelical (tertiary) structural elements are formed correctly as collapse proceeds. However, misfolding and non-obligatory intermediates are likely to occur and slow down overall folding. Thus, an essential element for fast folding is elimination of non-obligatory intermediates that can act as traps so that only the fast track is present. For helical proteins that are less well optimized, the kinetic intermediates change from non-obligatory to obligatory with the appearance of the disordered globule phase (Fig. 1b). In terms of
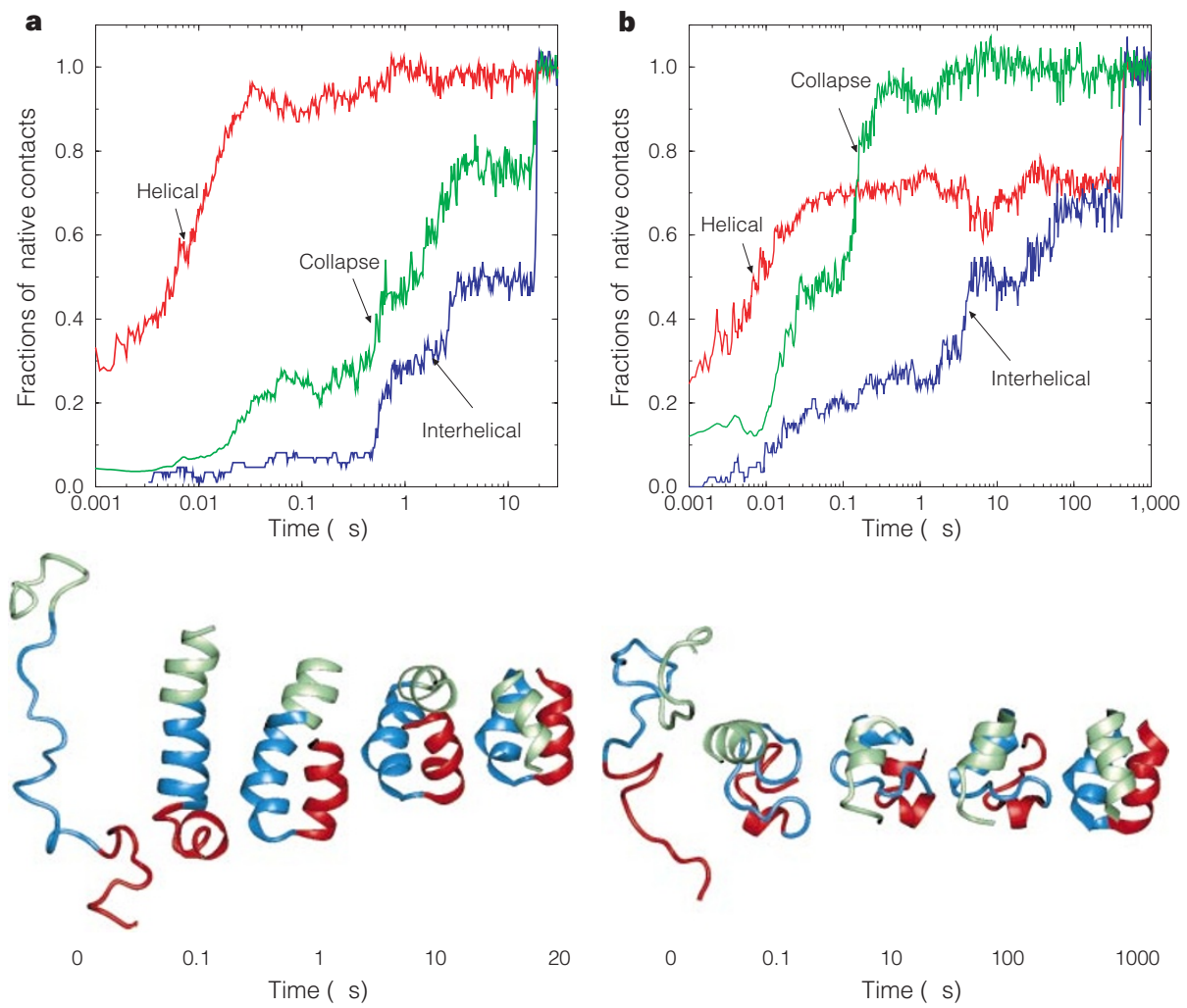

Figure 2 A semi-log plot of the time dependence of the fractions of native helical and interhelical contacts and inverse fraction of native volume (calculated from the inverse cube of the radius of gyration) for trajectories together with structures at selected times for

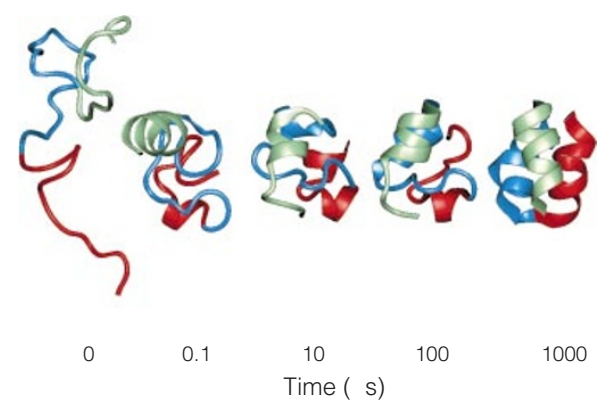

the $g=0.3$ model. $\mathbf{a}, \mathbf{b}$, Two individual trajectories; the final structure is the native structure. 


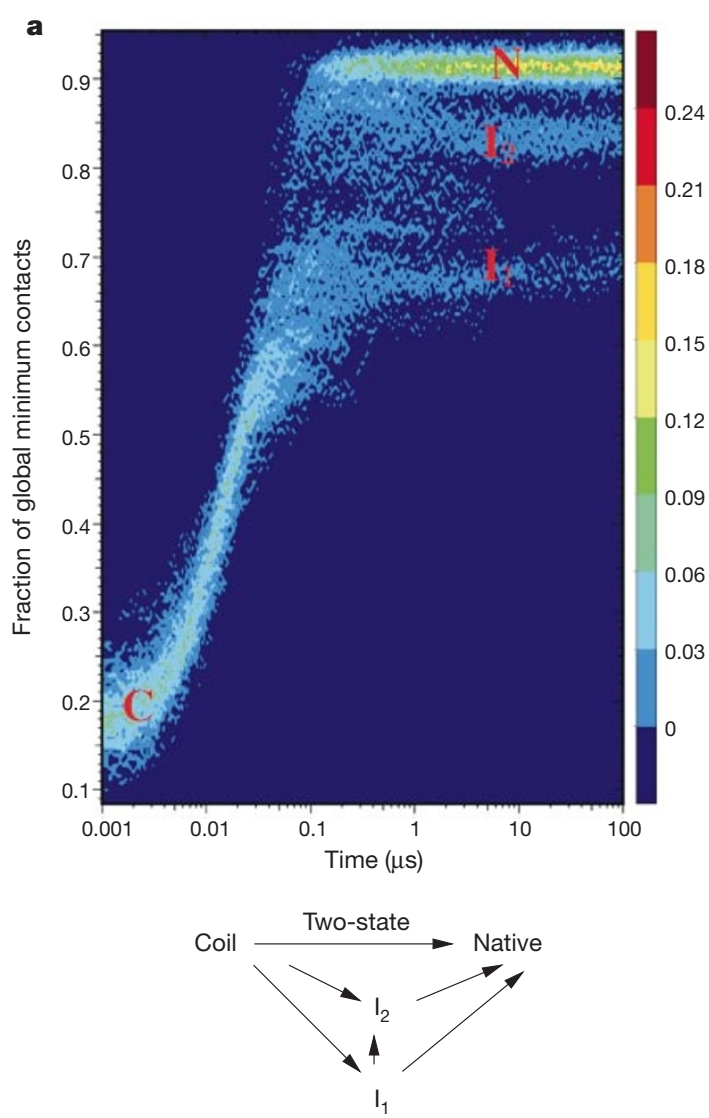

Figure 3 Folding mechanism of the large-gap ( $g=1.3$, a) and small-gap ( $g=0.3$, b) models. The distribution of the fraction of global minimum contacts is shown as a function of time (top). The distributions are obtained from independent kinetic simulations; the colour scale indicates the probabilities at given times. The various species ( $\mathrm{C}$, coil state; I, intermediates; $\mathrm{N}$, native state) that are important in folding pathways are indicated in red.

phenomenological descriptions, the folding changes from the diffusion-collision ${ }^{8}$ to simultaneous collapse and secondary structure formation with more local rearrangements to the native tertiary structure.

There have been many experimental studies of the folding of $\alpha$ helical proteins. The fast-folding $\lambda$-repressor $(0.2 \mathrm{~ms})^{23}$, which is well described by the diffusion-collision model, corresponds to the fast track in the large gap limit. By contrast, folding of apomyoglobin $^{24}$ involves an obligatory intermediate without a precise structure and folds more slowly $(0.5 \mathrm{~s})$, in accord with a small gap optimization. In four-helix-bundle immunity proteins ${ }^{25}$, the less stable (small gap) protein $(\operatorname{Im} 7)$ folds more slowly in the presence of kinetic intermediates, whereas the more stable (large gap) protein (Im9) folds via faster two-state mechanism. These illustrative comparisons indicate the potential utility of the present model for understanding how folding scenarios are realized in specific $\alpha$-helical proteins. It will be interesting to investigate other protein topologies (all- $\beta, \alpha / \beta)$ by the same approach.

\section{Methods}

\section{Model}

The model ${ }^{18}$ consists of 46 beads, each representing an amino-acid residue. The interaction potential between two non-bonded beads $i$ and $j$ is given by a square-well or squareshoulder potential. A Go-like model ${ }^{26}$ is used with the square-well depth (or squareshoulder height) for a pair of residues equal to $B_{\mathrm{N}} \epsilon$ if the pair is in contact in the global minimum structure and $B_{0} \epsilon\left(B_{0}>B_{N}\right)$, otherwise. The 'bias gap' $g, g=1-B_{0} / B_{N}$, is a measure of the difference in stability between the global minimum (native) contacts and other contacts. For $g>1$, non-native contacts are repulsive $\left(B_{\mathrm{O}}>0\right)$. The energy scale is determined by the parameter $\epsilon$. The model differs from earlier work ${ }^{18}$ by the presence of a chirality bias toward right-handed helices. A potential for the pseudodihedral angle $\alpha_{\mathrm{i}}$

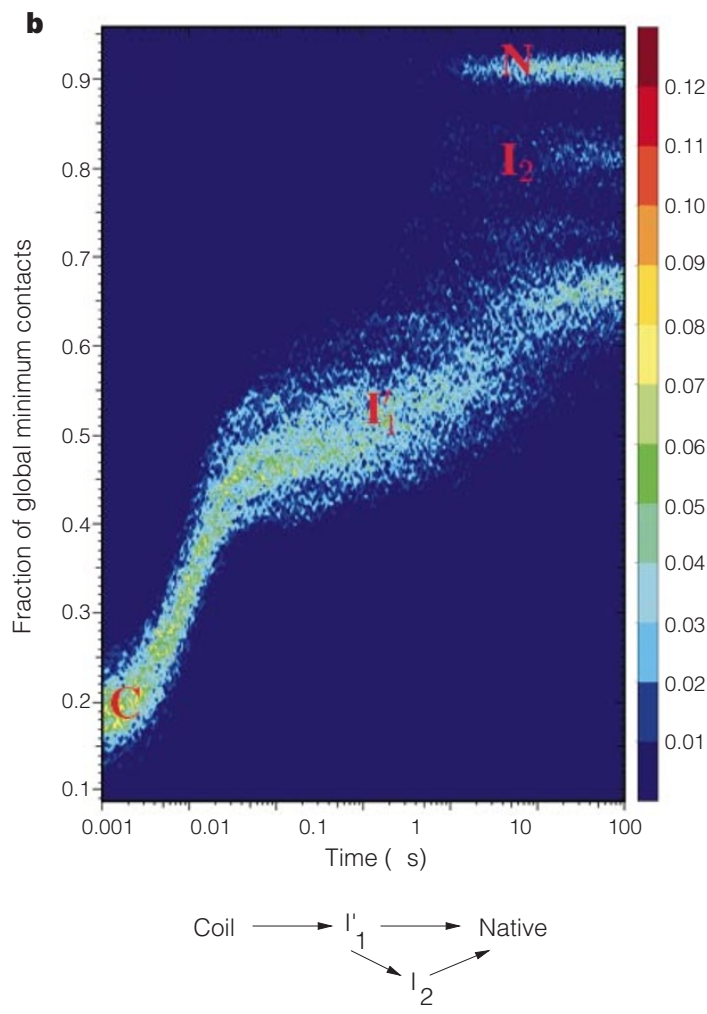

The folding pathways are shown below. For the large-gap model ( $g=1.3$, a), folding involves long-lasting non-obligatory intermediates $\left(l_{1}\right.$ and $\left.I_{2}\right)$, whereas for the small-gap model $(g=0.3, \mathbf{b})$, both obligatory on-pathway $\left(l_{1}^{\prime}\right)$ and non-obligatory $\left(l_{2}\right)$ intermediates are present.

between the corresponding $\mathrm{C}_{\alpha}$ atoms $\mathrm{i}, \mathrm{i}+1, \mathrm{i}+2$ and $\mathrm{i}+3$ is introduced. It has an energy $\epsilon_{\mathrm{b}}(=4 \epsilon)$ for $-180<\alpha_{\mathrm{i}}<0$ and 0 for $0<\alpha_{\mathrm{i}}<180$. The dihedral potential is applied to the $\alpha_{\mathrm{i}}$ that are positive (right-handed) in the global minimum structure.

\section{Thermodynamics}

The thermal transition temperatures in the phase diagram are based on heat-capacity maxima, which are obtained by the weighted-histogram method ${ }^{27}$ and constant-temperature discrete molecular dynamics ${ }^{28}$ at 17 different temperatures; five independent equilibrium simulations with 20-200-million collision steps each were conducted at every temperature. The collapse transition temperature is determined from the temperature at which the temperature derivative of the radius gyration square is a maximum (based on a spline fit).

\section{Kinetics}

Discrete molecular dynamics were used ${ }^{28}$. The 100 independent kinetic folding simulations were started with the equilibrated coil state at $T^{\star}=2.5$. The folding kinetics were investigated by quenching the temperature to $T^{*}=0.24$. A physical time scale was obtained by setting the average collapse time $(t \approx 1,000$ reduced time units) to $1 \mu$ s, the observed time for collapse of proteins ${ }^{29,30}$.

Received 26 April; accepted 29 July 1999.

1. Fersht, A. Enzyme Structure, Mechanism \& Protein Folding 3rd edn (W.H. Freeman, New York, 1998).

2. Dobson, D. M., Šali, A. \& Karplus, M. Protein folding: A perspective from theory and experiment. Angew. Chem. Int. Ed. 37, 868-893 (1998)

3. Šali, A. 100,000 protein structures for the biologist. Nature Struct. Biol. 5, 1029-1032 (1998).

4. Karplus, M. The Levinthal paradox: yesterday and today. Fold. Des. 2, 569-576 (1997).

5. Karplus, M. \& Šali, A. Theoretical studies of protein folding and unfolding. Curr. Opin. Struct. Biol. 5, 58-73 (1995).

6. Dill, K. A. \& Chan, H. S. From Levinthal to pathways to funnels. Nature Struct. Biol. 4, 10-19 (1997).

7. Socci, N. D., Onuchic, J. N. \& Wolynes, P. G. Protein folding mechanisms and the multidimensional folding funnel. Proteins 32, 136-158 (1998).

8. Karplus, M. \& Weaver, D. L. Protein-folding dynamics. Nature 260, 404-406 (1976).

9. Li, A. \& Daggett, V. Characterization of the transition state of protein unfolding by use of molecular dynamics: chymotrypsin inhibitor 2. Proc. Natl Acad. Sci. USA 91, 10430-10434 (1994). 
10. Caflisch, A. \& Karplus, M. Acid and thermal denaturation of Barnase investigated by molecular dynamics simulations. J. Mol. Biol. 252, 672-708 (1995).

11. Boczko, E. M. \& Brooks, C. L. III. First principles calculation of the folding free energy of a three-helix bundle protein. Science 269, 393-396 (1995).

12. Guo, Z. Y., Brooks, C. L. III. \& Boczko, E. M. Exploring the folding free energy surface of a three-helix bundle protein. Proc. Natl Acad. Sci. USA 94, 10161-10166 (1997).

13. Duan, Y. \& Kollman, P. A. Pathways to a protein folding intermediate observed in a 1-microsecond simulation in aqueous solution. Science 282, 707-744 (1998).

14. Gouda, H. et al. Three-dimensional solution structure of the B domain of Staphylococcal protein A Comparisons of the solution and crystal structures. Biochemistry 31, 9665-9672 (1992).

15. Zhou, Y., Vitkup, D. \& Karplus, M. Native proteins are surface-molten solids: Application of the Lindemann criterion for the solid versus liquid state. J. Mol. Biol. 285, 1371-1375 (1999).

16. Ptitsyn, O. B. Molten globule and protein folding. Adv. Protein Chem. 47, 83-230 (1995)

17. Privalov, P. L. Stability of proteins: Small globular proteins. Adv. Protein Chem. 33, 167-241 (1979).

18. Zhou, Y. \& Karplus, M. Folding thermodynamics of a model three-helix bundle protein. Proc. Nat Acad. Sci. USA 94, 14429-14432 (1997).

19. Schlunegger, M., Bennett, M. \& Eisenberg, D. Oligomer formation by 3D domain swapping: A model for protein assembly and misassembly. Adv. Protein Chem. 50, 61-122 (1997).

20. Lazaridis, T. \& Karplus, M. Multiple unfolding simulations reconcile the "new view" of protein folding with the old. Science 278, 1928-1931 (1997).

21. Roder, H. \& Colon, W. Kinetic role of early intermediates in protein folding. Curr. Opin. Struct. Biol. 7 , 15-28 (1997).

22. Frauenfelder, H. \& McMahon, B. Dynamics and function of proteins: The search for general concepts Proc. Natl Acad. Sci. USA 95, 4795-4797 (1998).

23. Burton, R. E., Myers, J. K. \& Oas, T. G. Protein folding dynamics—quantitative comparison between theory and experiment. Biochemistry 37, 5337-5343 (1998).

24. Cavagnero, S., Dyson, H. J. \& Wright, P. E. Effect of $\mathrm{H}$ helix destabilizing mutations on the kinetic and equilibrium folding of apomyoglobin. J. Mol. Biol. 285, 269-282 (1999)
25. Ferguson, N., Capaldi, A. P., James, R., Kleanthous, C. \& Radford, S. E. Rapid folding with and without populated intermediates in the homologous four-helix proteins $\operatorname{Im} 7$ and $\operatorname{Im} 9 . \mathrm{J}$. Mol. Biol. 286, 1597-1608 (1999).

26. Taketomi, H., Ueda, Y. \& Gō, N. Studies on protein folding, unfolding and fluctuations by computer simulations. Int. J. Pept. Protein Res. 7, 445-459 (1975).

27. Ferrenberg, A. M. \& Swendsen, R. H. Optimized Monte Carlo data analysis. Phys. Rev. Lett. 63, 1195 1197 (1989)

28. Zhou, Y., Karplus, M., Wichert, J. M. \& Hall, C. K. Equilibrium thermodynamics of homopolymers and clusters: Molecular dynamics and Monte Carlo simulations of systems with square-well interactions. J. Chem. Phys. 107, 10691-10708 (1997).

29. Ballew, R. M., Sabelko, J. \& Gruebele, M. Direct observation of fast protein folding: The initial collapse of apomyoglobin. Proc. Natl Acad. Sci. USA 93, 5759-5764 (1996).

30. Munoz, V., Thompson, P. A., Hofrichter, J. \& Eaton, W. A. Folding dynamics and mechanism of $\beta$ hairpin formation. Nature 390, 196-199 (1997).

\section{Acknowledgements}

We thank Y. Duan and P. A. Kollman for sending us the structure of the intermediate observed in their trajectory. This work was supported in part by a grant from the NSF and grant from Pittsburgh Supercomputing Center, and from UC Berkeley Network of

Workstations (NOW) through NAPCI (National Partnership for Advanced Computational Infrastructure). Y.Z. is an NIH postdoctoral fellow. The calculations at Harvard were conducted on HP 9000/735, DEC Alpha and SUN UltraSparc workstations. The drawing of the three-helix bundle were made with QUANTA (Molecular Simulations Inc.).

Correspondence and requests for materials should be addressed to M.K. (e-mail: marci@tammy.harvard. edu).

\section{errata}

\section{Selective inhibition of cocaine- seeking behaviour by a partial dopamine $\mathrm{D}_{3}$ receptor agonist}

Maria Pilla, Sylvie Perachon, François Sautel, Fabrice Garrido, André Mann, Camille G. Wermuth, Jean-Charles Schwartz, Barry J. Everitt \& Pierre Sokoloff

Nature 400, 371-375 (1999)

Spurious symbols were introduced into Fig. 1d during reproduction. The correct figure is shown here.
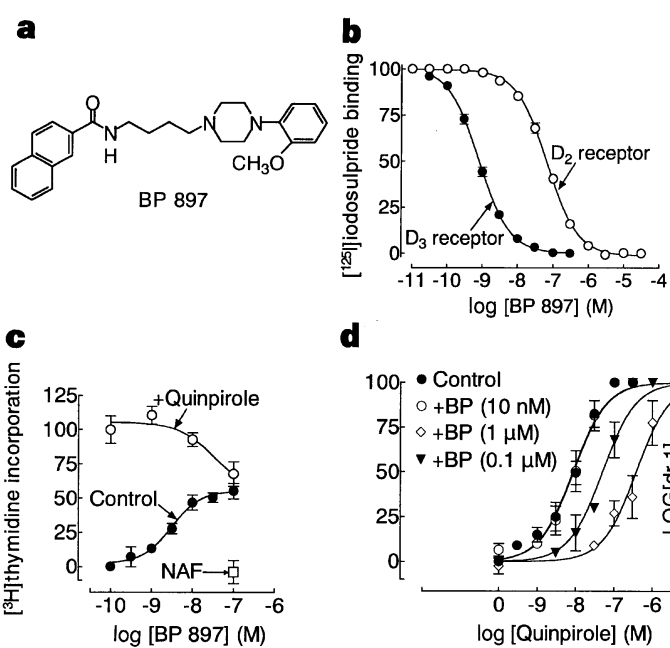

d

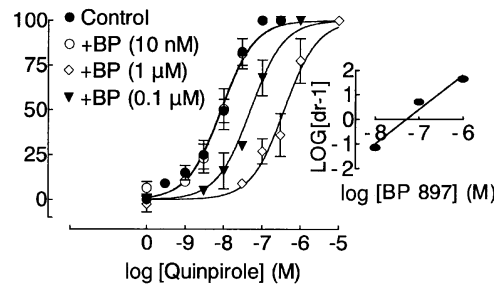

\section{Binding of double-strand breaks in DNA by human Rad52 protein}

\section{Eric Van Dyck, Alicja Z. Stasiak, Andrzej Stasiak} \& Stephen C. West

Nature 398, 728-731 (1999)

In Fig. 4, one of the strands of duplex DNA was deleted. The corrected figure is shown below.

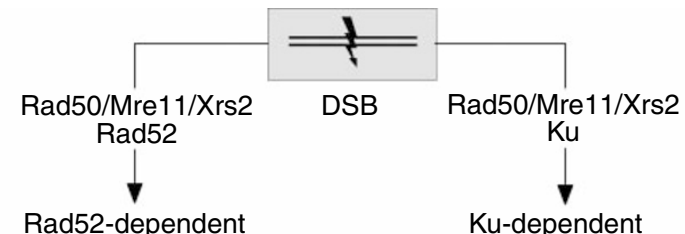

homologous recombination

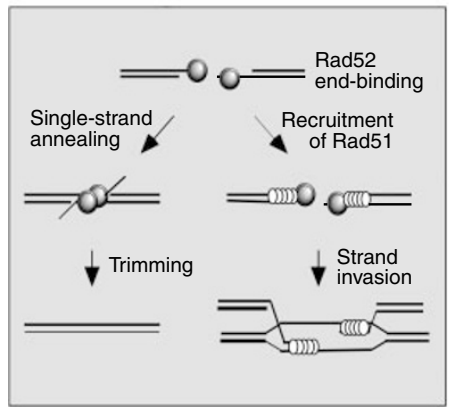

non-homologous end-joining

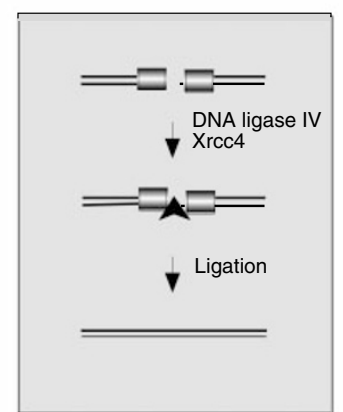

